\title{
Stepsize Restrictions for Nonlinear Stability Properties of Neutral Delay Differential Equations
}

\author{
Wei Gu, ${ }^{1}$ Ming Wang, ${ }^{2}$ and Dongfang $\mathrm{Li}^{3}$ \\ ${ }^{1}$ School of Statistics and Mathematics, Zhongnan University of Economics and Law, Wuhan 430073, China \\ ${ }^{2}$ School of Mathematics and Physics, China University of Geosciences, Wuhan 430074, China \\ ${ }^{3}$ School of Mathematics and Statistics, Huazhong University of Science and Technology, Wuhan 430074, China \\ Correspondence should be addressed to Dongfang Li; hustldf@gmail.com
}

Received 22 May 2014; Accepted 9 July 2014; Published 21 July 2014

Academic Editor: Ali H. Bhrawy

Copyright (C) 2014 Wei Gu et al. This is an open access article distributed under the Creative Commons Attribution License, which permits unrestricted use, distribution, and reproduction in any medium, provided the original work is properly cited.

The present paper is concerned with the relationship between stepsize restriction and nonlinear stability of Runge-Kutta methods for delay differential equations. We obtain a special stepsize condition guaranteeing global and asymptotical stability properties of numerical methods. Some confirmations of the conditions on Runge-Kutta methods are illustrated at last.

\section{Introduction}

Neutral delay differential equations (NDDEs) are widely used in various kinds of applied disciplines such as biology, ecology, electrodynamics, and physics and hence intrigue lots of researchers in numerical simultation and analysis (see, e.g., [1-3]). Up to now, many researchers have discussed nonlinear stability properties for NDDEs. In 2000, Bellen et al. [4] studied $B N_{f}$-stable continuous Runge-Kutta methods for NDDEs. They extended the contractivity requirements to the numerical stability analysis. Vermiglio and Torelli further pointed out that the numerical solution produced by the methods can preserve the contractivity property of the theoretical solution in [5]. In 2002, Zhang [6] derived nonlinear stability properties for theoretical and numerical solutions of NDDEs based on natural Runge-Kutta schemes. After that, Wang et al. [7,8] first introduced the concepts of GS(l)- and GAS $(l)$-stability for nonautonomous nonlinear problems. They proved that $(k, l)$-algebraically stable RungeKutta methods and $(k, p, 0)$-algebraically stable general linear methods lead to GS(l)- and GAS $(l)$-stability for NDDEs, respectively. Recently, Bhrawy et al. [9-11] studied several kinds of collocation method for some NDDEs. For more analogues results, we refer readers to [12-15]. Useful as these stability results are, however, no conclusions have been found to develop the relationship between nonlinear stability analysis and stepsize restriction with some numerical schemes for NDDEs, especially for some Runge-Kutta methods.

The present paper was in part inspired by the work of Spijker et al. With stepsize restriction to some numerical schemes, they revealed to us some monotonicity and stability properties for ODEs, respectively (see, [16-19]). We extend their study to nonlinear NDDEs in the present paper. With stepsize restriction to Runge-Kutta schemes, global and asymptotical stability results for NDDEs are obtained, respectively.

The rest of the paper is organized as follows. In Section 2, we consider Runge-Kutta schemes with linear interpolation procedure for NDDEs. Some concepts, such as global and asymptotical stability, are also collected. Section 3 is devoted to stability analysis. The given results set up a relationship between the stepsize restriction and nonlinear stability for nonlinear NDDEs. Some examples of Runge-Kutta schemes are presented in Section 4. Finally, we end up with some conclusions and extension in the last section.

\section{Runge-Kutta Methods for NDDEs}

In the present paper, we consider the following nonlinear NDDEs:

$$
\frac{d}{d t}[y(t)-N y(t-\tau)]=f(t, y(t), y(t-\tau)), \quad t>0,
$$




$$
y(t)=\psi(t), \quad-\tau \leq t \leq 0
$$

and the perturbed problem

$$
\begin{gathered}
\frac{d}{d t}[z(t)-N z(t-\tau)]=f(t, z(t), z(t-\tau)), \quad t>0, \\
z(t)=\phi(t) \quad-\tau \leq t \leq 0 .
\end{gathered}
$$

Here, $\tau$ denotes a positive delay term, $N \in \mathbb{C}^{d \times d}$ is a constant matrix with $\|N\|<1, \psi(t)$ and $\phi(t)$ are continuous, and $f:[0,+\infty] \times X \times X \rightarrow X$, such that (1) and (2) own a unique solution, respectively, where $X$ is a real or complex Hilbert space. As in $[20,21]$, we assume there exist some inner product $\langle\cdot, \cdot\rangle$ and the induced norm $\|\cdot\|$ such that

$$
\begin{aligned}
\operatorname{Re}\langle(y-z)-N(u-v), f(t, y, u)-f(t, z, v)\rangle \\
\leq \alpha\|y-z\|^{2}+\beta\|u-v\|^{2} \\
\quad+\delta\|f(t, y, u)-f(t, z, v)\|^{2},
\end{aligned}
$$

where $\alpha \leq 0, \beta \geq 0$, and $\delta<0$ are real constants.

When $N=0$, the problem (1) degenerates into nonlinear DDEs of the following type:

$$
\begin{gathered}
y^{\prime}(t)=f(t, y(t), y(t-\tau)), \quad t>0, \\
y(t)=\psi(t) \quad-\tau \leq t \leq 0 .
\end{gathered}
$$

Nonlinear stability analysis for such systems can be found in $[6,22-25]$. Condition (3) can be equivalent to the assumptions in these literatures (see [26], Remark 2.1).

Now, let us consider s-stage Runge-Kutta methods for (1); the coefficients of the schemes may be organized in Buther tableau as follows:

$$
\begin{array}{c|c}
c & A \\
\hline & b^{T}
\end{array}
$$

where $c=\left[c_{l}, \ldots, c_{s}\right]^{T}, b=\left[b_{1}, \ldots, b_{s}\right]^{T}$, and $A=\left(a_{i j}\right)_{i, j=1}^{s}$.

According to Liu in [27], Runge-Kutta methods for NDDEs can be written as

$$
\begin{aligned}
& y_{n+1}-N \tilde{y}_{n+1} \\
& =y_{n}-N \tilde{y}_{n}+h \sum_{j=1}^{s} b_{j} f\left(t_{n}+c_{j} h, y_{j}^{(n)}, \widetilde{y}_{j}^{(n)}\right), \\
& y_{i}^{(n)}-N \tilde{y}_{i}^{(n)} \\
& =y_{n}-N \tilde{y}_{n}+h \sum_{j=1}^{s} a_{i j} f\left(t_{n}+c_{j} h, y_{j}^{(n)}, \widetilde{y}_{j}^{(n)}\right) \\
& \quad i=1,2, \ldots, s,
\end{aligned}
$$

where $h$ is stepsize and $t_{n}=n h, y_{n}, \tilde{y}_{n}, y_{i}^{(n)}$ and $\widetilde{y}_{i}^{(n)}$ are approximations to the analytic solutions $y\left(t_{n}\right), y\left(t_{n}-\tau\right)$, $y\left(t_{n}+c_{i} h\right)$, and $y\left(t_{n}+c_{i} h-\tau\right)$, respectively. We set $\tau=(m-\theta) h$ with $\theta \in[0,1)$, and the arguments $\widetilde{y}_{n}$ and $\widetilde{y}_{j}^{(n)}$ are determined by

$$
\begin{gathered}
\tilde{y}_{n}=\theta y_{n-m+1}+(1-\theta) y_{n-m}, \\
\widetilde{y}_{j}^{(n)}=\theta y_{j}^{(n-m+1)}+(1-\theta) y_{j}^{(n-m)},
\end{gathered}
$$

where $y_{i}=\psi\left(t_{i}\right)$ for $t_{i} \leq 0$ and $y_{j}^{(i)}=\psi\left(t_{i}+c_{j} h\right)$ for $t_{i}+c_{j} h \leq 0$.

Now, let $y_{n}$ and $z_{n}$ be two sequences of approximations to problems (1) and (2), respectively. Following Definitions 9.1.1 and 9.1.2 in [1] for delay systems, we introduce some stability concepts.

Definition 1. A numerical method for DDEs or NDDEs is called globally stable, if there exists a constant $C$ such that

$$
\left\|y_{n}-z_{n}\right\| \leq C \max _{-\tau \leq t \leq 0}\|\psi(t)-\phi(t)\|
$$

holds when the method is applied to (1) and (2) under some assumptions.

Definition 2. A numerical method for DDEs or NDDEs is said to be asymptotically stable, if

$$
\lim _{n \rightarrow \infty}\left\|y_{n}-z_{n}\right\|=0
$$

holds when the method is applied to (1) and (2) under some assumptions.

\section{Stability Analysis}

In the section, we will discuss the relationship between the stepsize restriction and nonlinear stability of the method.

Theorem 3. Assume condition (3) holds, $\alpha+\beta \leq 0$, and there exists a positive real number $r$, such that the matrix

$$
M=\operatorname{diag}(b) A+A^{T} \operatorname{diag}(b)-b b^{T}+\frac{1}{r} \operatorname{diag}(b)
$$

is nonnegative definite, where $b_{i} \geq 0, i=1,2, \ldots, s$. Then the Runge-Kutta method with linear interpolation procedure for NDDEs (1) is globally stable under the stepsize restriction

$$
\frac{h}{r} \leq-2 \delta
$$

Proof. Let $\left\{y_{n}, y_{i}^{(n)}, \tilde{y}_{i}^{(n)}\right\}$ and $\left\{z_{n}, z_{i}^{(n)}, \widetilde{z}_{i}^{(n)}\right\}$ be two sequences of approximations to problems (1) and (2), respectively, and write

$$
\begin{gathered}
U_{i}^{(n)}=y_{i}^{(n)}-z_{i}^{(n)}, \quad \widetilde{U}_{0}^{(n)}=\widetilde{y}_{i}^{(n)}-\widetilde{z}_{i}^{(n)}, \\
U_{0}^{(n)}=y_{n}-z_{n}, \quad \widetilde{U}_{0}^{(n)}=\widetilde{y}_{n}-\widetilde{z}_{n}, \\
W_{i}=h\left[f\left(t_{n}+c_{i} h, y_{i}^{(n)}, \widetilde{y}_{i}^{(n)}\right)-f\left(t_{n}+c_{i} h, z_{i}^{(n)}, \widetilde{z}_{i}^{(n)}\right)\right] .
\end{gathered}
$$


With the notation, Runge-Kutta methods with the same stepsize $h$ for (1) and (2) yield

$$
\begin{gathered}
U_{0}^{(n+1)}-N \widetilde{U}_{0}^{(n+1)}=U_{0}^{(n)}-N \widetilde{U}_{0}^{(n)}+\sum_{j=1}^{s} b_{j} W_{j}, \\
U_{i}^{(n)}-N \widetilde{U}_{i}^{(n)}=U_{0}^{(n)}-N \widetilde{U}_{0}^{(n)}+\sum_{j=1}^{s} a_{i j} W_{j}, \quad i=1,2, \ldots, s .
\end{gathered}
$$

Thus, we have

$$
\begin{aligned}
\left\|U_{0}^{(n+1)}-N \widetilde{U}_{0}^{(n+1)}\right\|^{2} & \left\langle U_{0}^{(n)}-N \widetilde{U}_{0}^{(n)}+\sum_{j=1}^{s} b_{j} W_{j}, U_{0}^{(n)}-N \widetilde{U}_{0}^{(n)}+\sum_{i=1}^{s} b_{i} W_{i}\right\rangle \\
= & \left\|U_{0}^{(n)}-N \widetilde{U}_{0}^{(n)}\right\|^{2}+2 \sum_{i=1}^{s} b_{i} \operatorname{Re}\left\langle U_{0}^{(n)}-N \widetilde{U}_{0}^{(n)}, W_{i}\right\rangle \\
& +\sum_{i, j=1}^{s} b_{i} b_{j}\left\langle W_{i}, W_{j}\right\rangle \\
= & \left\|U_{0}^{(n)}-N \widetilde{U}_{0}^{(n)}\right\|^{2} \\
& +2 \sum_{i=1}^{s} b_{i} \operatorname{Re}\left\langle U_{i}^{(n)}-N \widetilde{U}_{i}^{(n)}-\sum_{j=1}^{s} a_{i j} W_{j}, W_{i}\right\rangle \\
& +\sum_{i, j=1}^{s} b_{i} b_{j}\left\langle W_{i}, W_{j}\right\rangle \\
= & \left\|U_{0}^{(n)}-N \widetilde{U}_{0}^{(n)}\right\|^{2}+2 \sum_{i=1}^{s} b_{i} \operatorname{Re}\left\langle U_{i}^{(n)}-N \widetilde{U}_{i}^{(n)}, W_{i}\right\rangle \\
& -\sum_{i, j=1}^{s}\left(b_{i} a_{i j}+a_{j i} b_{j}-b_{i} b_{j}\right)\left\langle W_{i}, W_{j}\right\rangle .
\end{aligned}
$$

Now, in view of the nonnegative definite matrix $M$, we obtain

$$
-\sum_{i, j=1}^{s}\left(b_{i} a_{i j}+a_{j i} b_{j}-b_{i} b_{j}\right)\left\langle W_{i}, W_{j}\right\rangle \leq \frac{1}{r} \sum_{i=1}^{s} b_{i}\left\langle W_{i}, W_{i}\right\rangle .
$$

On the other hand, in terms of condition (3), we find

$$
\begin{aligned}
& \operatorname{Re}\left\langle U_{i}^{(n)}-N \widetilde{U}_{i}^{(n)}, W_{i}\right\rangle \\
& \quad \leq \alpha h\left\|U_{i}^{(n)}\right\|^{2}+\beta h\left\|\widetilde{U}_{i}^{(n)}\right\|^{2}+\frac{\delta}{h}\left\|W_{i}\right\|^{2} .
\end{aligned}
$$

Then, together with (14), (15), and (16) and using the conditions $h / r \leq-2 \delta$, we get

$$
\begin{aligned}
& \left\|U_{0}^{(n+1)}-N \widetilde{U}_{0}^{(n+1)}\right\|^{2} \\
& \leq\left\|U_{0}^{(n)}-N \widetilde{U}_{0}^{(n)}\right\|^{2} \\
& \quad+2 \sum_{i=1}^{s} h b_{i}\left(\alpha h\left\|U_{i}^{(n)}\right\|^{2}+\beta h\left\|\widetilde{U}_{i}^{(n)}\right\|^{2}+\left(\frac{\delta}{h}+\frac{1}{2 r}\right)\left\|W_{i}\right\|^{2}\right) \\
& \leq\left\|U_{0}^{(n)}-N \widetilde{U}_{0}^{(n)}\right\|^{2}+2 \sum_{i=1}^{s} h b_{i}\left(\alpha\left\|U_{i}^{(n)}\right\|^{2}+\beta\left\|\widetilde{U}_{i}^{(n)}\right\|^{2}\right) .
\end{aligned}
$$

Noting that

$$
\begin{aligned}
\left\|\widetilde{U}_{i}^{(n)}\right\|^{2}= & {\left[\theta\left\|U_{i}^{(n-m+1)}\right\|+(1-\theta)\left\|U_{i}^{(n-m)}\right\|\right]^{2} } \\
\leq & \theta^{2}\left\|U_{i}^{(n-m+1)}\right\|^{2}+(1-\theta)^{2}\left\|U_{i}^{(n-m)}\right\|^{2} \\
& +\theta(1-\theta)\left(\left\|U_{i}^{(n-m+1)}\right\|^{2}+\left\|U_{i}^{(n-m)}\right\|^{2}\right) \\
= & \theta\left\|U_{i}^{(n-m+1)}\right\|^{2}+(1-\theta)\left\|U_{i}^{(n-m)}\right\|^{2}
\end{aligned}
$$

and $\alpha+\beta \leq 0$, we have

$$
\begin{aligned}
& \left\|U_{0}^{(n+1)}-N \widetilde{U}_{0}^{(n+1)}\right\|^{2} \\
& \leq\left\|U_{0}^{(n)}-N \widetilde{U}_{0}^{(n)}\right\|^{2}+2 \sum_{i=1}^{s} h \beta b_{i}\left(\left\|\widetilde{U}_{i}^{(n)}\right\|^{2}-\left\|U_{i}^{(n)}\right\|^{2}\right) \\
& \leq\left\|U_{0}^{(n)}-N \widetilde{U}_{0}^{(n)}\right\|^{2} \\
& \quad+2 \sum_{i=1}^{s} h \beta b_{i}\left(\theta\left\|U_{i}^{(n-m+1)}\right\|^{2}\right. \\
& \leq\left\|U_{0}^{(0)}-N \widetilde{U}_{0}^{(0)}\right\|^{2}+2 \sum_{i=1}^{s} h \beta b_{i}\left(\sum_{j=-m+1}^{-1} \theta\left\|U_{i}^{(j)}\right\|^{2}\right. \\
& \leq\left(2+2\|N\|^{2}+2 \tau \sum_{i=1}^{s} \beta b_{i}\right) \max _{-\tau \leq t \leq 0}\|\psi(t)-\phi(t)\|^{2} \\
& \left.\leq \quad 2\left\|U_{0}^{(0)}\right\|^{2}+2\|N\|\left\|\widetilde{U}_{0}^{(0)}\right\|^{2}+2 \sum_{i=1}^{s} m h \beta b_{i}^{(n-m)}\left\|_{-m \leq j \leq-1}^{2}-\right\| U_{i}^{(n)} \|^{2}\right) \\
& \left.\leq\left\|U_{0}^{(0)}-N \widetilde{U}_{0}^{(0)}\right\|^{2}+2 \sum_{j=-m}^{s} m h \beta b_{i} \max _{-m \leq j \leq-1}\left\|U_{i}^{(j)}\right\|^{2}(1-\theta)\left\|U_{i}^{(j)}\right\|^{2}\right)
\end{aligned}
$$


This implies that

$$
\left\|U_{0}^{(n+1)}-N \widetilde{U}_{0}^{(n+1)}\right\| \leq \widetilde{C} \max _{-\tau \leq t \leq 0}\|\psi(t)-\phi(t)\|,
$$

where $\widetilde{C}=\sqrt{\left(2+2\|N\|^{2}+2 \tau \beta\right)}$.

Note that $\|N\|<1$; we have

$$
\begin{aligned}
\left\|U_{0}^{(n+1)}\right\| \leq & \|N\|\left\|\widetilde{U}_{0}^{(n+1)}\right\|+\widetilde{C} \max _{-\tau \leq t \leq 0}\|\psi(t)-\phi(t)\| \\
= & \|N\|\left\|\theta U_{0}^{n-m+2}+(1-\theta) U_{0}^{n-m+1}\right\| \\
& +\widetilde{C} \max _{-\tau \leq t \leq 0}\|\psi(t)-\phi(t)\| \\
\leq & \max \left(\left\|U_{0}^{n-m+2}\right\|,\left\|U_{0}^{n-m+1}\right\|\right) \\
& +\widetilde{C} \max _{-\tau \leq t \leq 0}\|\psi(t)-\phi(t)\| .
\end{aligned}
$$

An induction to (21) yields

$$
\left\|U_{0}^{(n+1)}\right\| \leq(1+\widetilde{C}) \max _{-\tau \leq t \leq 0}\|\psi(t)-\phi(t)\| .
$$

Therefore, the conclusion is proven.

Corollary 4. Assume condition (3) holds; $\alpha+\beta \leq 0$. Then an algebraically stable Runge-Kutta method with linear interpolation procedure for DDEs (4) or NDDEs (1) is globally stable.

Remark 5. A Runge-Kutta method is algebraically stable if the matrix

$$
\operatorname{diag}(b) A+A^{T} \operatorname{diag}(b)-b b^{T}
$$

is nonnegative definite and $b_{i} \geq 0(i=1,2, \ldots, s)$. For example, the $s$-stage Gauss, Radau I $A$, Radau II $A$, and Lobatto IIIC methods are algebraically stable. Corollary 4 can be derived for $r=\infty$. This implies that the stepsize restriction for DDEs disappears.

Corollary 6. Assume condition (3) holds, $\alpha+\beta \leq 0$, and there exists a positive real number $r$, such that the matrix

$$
M=\operatorname{diag}(b) A+A^{T} \operatorname{diag}(b)-b b^{T}+\frac{1}{r} \operatorname{diag}(b)
$$

is nonnegative definite, where $b_{i} \geq 0, i=1,2, \ldots, s$. Then the Runge-Kutta method with linear interpolation procedure for DDEs (4) is globally stable under the stepsize restriction

$$
\frac{h}{r} \leq-2 \delta
$$

Theorem 7. Assume condition (3) holds, $\alpha+\beta<0$, the function $f(t, u, v)$ is uniformly Lipschitz continuous with constant $L$ in variables $u$ and $v$, and there exists a positive real number $r$, such that the matrix

$$
M=\operatorname{diag}(b) A+A^{T} \operatorname{diag}(b)-b b^{T}+\frac{1}{r} \operatorname{diag}(b)
$$

is nonnegative definite, where $b_{i} \geq 0, i=1,2, \ldots, s$. Then the Runge-Kutta method with linear interpolation procedure for NDDEs (1) is asymptotically stable under the stepsize restriction

$$
\frac{h}{r} \leq-2 \delta
$$

Proof. Like in the proof of Theorem 3, let $\sigma=\alpha+\beta<0$, and we can easily find

$$
\begin{aligned}
\left\|U_{0}^{(n+1)}-N \widetilde{U}_{0}^{(n+1)}\right\|^{2} & \\
\leq & \left\|U_{0}^{(n)}-N \widetilde{U}_{0}^{(n)}\right\|^{2}+2 \sum_{i=1}^{s} h b_{i}\left(\alpha\left\|U_{i}^{(n)}\right\|^{2}+\beta\left\|\widetilde{U}_{i}^{(n)}\right\|^{2}\right) \\
\leq & 2\left(\left\|U_{0}^{(n)}\right\|^{2}+\|N\|^{2}\left\|\widetilde{U}_{0}^{(n)}\right\|^{2}\right) \\
& +2 \sum_{i=1}^{s} h b_{i}\left((\sigma-\beta)\left\|U_{i}^{(n)}\right\|^{2}+\beta\left\|\widetilde{U}_{i}^{(n)}\right\|^{2}\right) \\
= & 2\left(\left\|U_{0}^{(n)}\right\|^{2}+\|N\|^{2}\left\|\widetilde{U}_{0}^{(n)}\right\|^{2}\right) \\
& +2 \sum_{i=1}^{s} h \beta b_{i}\left(\left\|\widetilde{U}_{i}^{(n)}\right\|^{2}-\left\|U_{i}^{(n)}\right\|^{2}\right)+2 \sum_{i=1}^{s} h b_{i} \sigma\left\|U_{i}^{(n)}\right\|^{2} \\
\leq & \left(2+2\|N\|^{2}+2 \tau \sum_{i=1}^{s} \beta b_{i}\right) \max _{-\tau \leq t \leq 0}\|\psi(t)-\phi(t)\|^{2} \\
& +2 \sum_{j=1}^{n} \sum_{i=1}^{s} h b_{i} \sigma\left\|U_{i}^{(j)}\right\|^{2} .
\end{aligned}
$$

Note $\sigma<0$ and $b_{i} \geq 0$; we have

$$
\lim _{n \rightarrow \infty} \sum_{i=1}^{s} b_{i}\left\|U_{i}^{(n)}\right\|=0 .
$$

On the other hand,

$$
\begin{aligned}
\left\|W_{i}\right\| & =\left\|h\left[f\left(t_{n}+c_{i} h, y_{i}^{(n)}, \widetilde{y}_{i}^{(n)}\right)-f\left(t_{n}+c_{i} h, z_{i}^{(n)}, \widetilde{z}_{i}^{(n)}\right)\right]\right\| \\
& \leq h L\left(\left\|U_{i}^{(n)}\right\|+\left\|\widetilde{U}_{i}^{(n)}\right\|\right) .
\end{aligned}
$$

Now, in view of (13), (29), and (30), we obtain

$$
\lim _{n \rightarrow \infty}\left\|U_{0}^{(n)}-N \widetilde{U}_{0}^{(n)}\right\|=0 .
$$

Since

$$
\begin{aligned}
\left\|U_{0}^{(n)}\right\| & =\left\|U_{0}^{(n)}-N \widetilde{U}_{0}^{(n)}+N \widetilde{U}_{0}^{(n)}\right\| \\
& \leq\left\|U_{0}^{(n)}-N \widetilde{U}_{0}^{(n)}\right\|+\|N\|\left\|\widetilde{U}_{0}^{(n)}\right\| \\
& \leq\left\|U_{0}^{(n)}-N \widetilde{U}_{0}^{(n)}\right\|+\|N\| \max \left(\left\|U_{0}^{n-m+2}\right\|,\left\|U_{0}^{n-m+1}\right\|\right)
\end{aligned}
$$

and $\|N\|<1$, an induction to (32) gives

$$
\lim _{n \rightarrow \infty}\left\|U_{0}^{(n)}\right\|=0
$$

which completes the proof. 
Corollary 8. Assume condition (3) holds, $\alpha+\beta<0$, the function $f(t, u, v)$ is uniformly Lipschitz continuous with constant $L$ in variables $u$ and $v$. Then an algebraically stable Runge-Kutta method with linear interpolation procedure for DDEs (4) or NDDEs (1) is asymptotically stable.

Corollary 9. Assume condition (3) holds, $\alpha+\beta<0$, the function $f(t, u, v)$ is uniformly Lipschitz continuous with constant $L$ in variables $u$ and $v$, and there exists a positive real number $r$, such that the matrix

$$
M=\operatorname{diag}(b) A+A^{T} \operatorname{diag}(b)-b b^{T}+\frac{1}{r} \operatorname{diag}(b)
$$

is nonnegative definite, where $b_{i} \geq 0, i=1,2, \ldots, s$. Then the Runge-Kutta method with linear interpolation procedure for DDEs (4) is asymptotically stable under the stepsize restriction

$$
\frac{h}{r} \leq-2 \delta .
$$

\section{Some Examples}

As it is shown in the theorems, the parameters $\delta$ and $r$ in the matrix $M$ play a key role in the stability analysis. The larger the existed parameter $r$ is, the larger stepsize we could choose. In this section, we will show some examples.

Consider the following case, like the conditions in [22] or [28], if $f(t, y, u)=\tilde{f}(t, y-N u)$ and

$$
\begin{aligned}
& \|\rho((y-N u)-(z-N v))+\tilde{f}(t, y-N u)-\tilde{f}(t, z-N v)\| \\
& \quad \leq \rho\|((y-N u)-(z-N v))\|
\end{aligned}
$$

with $\rho>0$, we have the following form in an inner product norm:

$$
\begin{aligned}
\operatorname{Re} & \langle(y-N u)-(z-N v), \tilde{f}(t, y-N u)-\tilde{f}(t, z-N v)\rangle \\
& \leq \delta\|\tilde{f}(t, y-N u)-\tilde{f}(t, z-N v)\|^{2}
\end{aligned}
$$

with $\delta=-1 /(2 \rho)<0$.

In particular, let $f(t, y, u)=-a(M y-N u)$, where $a>$ $0, M<1$ are constants independent of $t$, respectively. We have

$$
\begin{aligned}
\operatorname{Re} & \langle y-N u, f(t, y, u)\rangle \\
= & \operatorname{Re}\langle M y-N u-(M-1) y,-a(M y-N u)\rangle \\
= & -\frac{1}{a}\|a(M y+N u)\|^{2}+a M(M-1)\|y\|^{2} \\
& +\operatorname{Re}\langle a(1-M) y, N u\rangle \\
\leq & -\frac{1}{a}\|a(M y+N u)\|^{2}+a M(M-1)\|y\|^{2}
\end{aligned}
$$

$$
\begin{aligned}
& +\frac{1}{2} a(1-M)\|N\|\left(\|y\|^{2}+\|u\|^{2}\right) \\
= & \left(a M(M-1)+\frac{1}{2} a(1-M)\|N\|\right)\|y\|^{2} \\
& +\frac{1}{2} a(1-M)\|N\|\|u\|^{2}-\frac{1}{a}\|f(t, y, u)\|^{2} .
\end{aligned}
$$

Next, we give some examples on how to calculate the parameter $r$.

Example 1. Consider $s$-stage 1-order Runge-Kutta methods (see [17], section 2.7)

$$
\begin{array}{c|cccccc}
0 & 0 & & & & \\
\frac{1}{s} & \frac{1}{s} & 0 & & & & \\
\frac{2}{2} & \frac{1}{s} & \frac{1}{s} & 0 & & & \\
\hline s & \frac{s}{s} & & & & \\
\vdots & \vdots & \vdots & \vdots & & & \\
\frac{s-1}{s} & \frac{1}{s} & \frac{1}{s} & \frac{1}{s} & \ldots & \frac{1}{s} & 0 \\
\hline & \frac{1}{s} & \frac{1}{s} & \frac{1}{s} & \frac{1}{s} & \frac{1}{s} & \frac{1}{s} \\
& \frac{s}{s} & \frac{s}{s} & \frac{s}{s} &
\end{array}
$$

and we have

$$
\begin{aligned}
M & =\operatorname{diag}(b) A+A^{T} \operatorname{diag}(b)-b b^{T}+\frac{1}{r} \operatorname{diag}(b) \\
& =\left(\frac{1}{r s}-\frac{1}{s^{2}}\right) I_{s} .
\end{aligned}
$$

Thus, the matrix $M$ is nonnegative definite for $0<r \leq s$. They imply that these methods for DDE with interpolation are stable with stepsize restriction $h \leq-2 \delta s$.

Example 2. Consider 2-stage 2-order Runge-Kutta method:

$$
\begin{array}{l|ll}
0 & 0 & 0 \\
1 & 1 & 0 \\
\hline & \frac{1}{2} & \frac{1}{2}
\end{array}
$$

and we obtain

$$
M=\left[\begin{array}{cc}
\frac{1}{2 r}-\frac{1}{4} & \frac{1}{4} \\
\frac{1}{4} & \frac{1}{2 r}-\frac{1}{4}
\end{array}\right] .
$$

Therefore, the matrix $M$ is nonnegative definite for $0<r \leq 1$. They imply that the stepsize $h \leq-2 \delta$ is feasible under the assumptions (3) for NDDEs (1).

For more Runge-Kutta methods with the nonnegative definite matrix $M$, we refer readers to Section 2.2.4 in [28]. Higueras revealed to us how to find the largest $r$ such that the matrix $M$ is nonnegative definite. He pointed that if 
the matrix $\operatorname{diag}(b)$ is positive definite, the largest $r$ can be determined by

$$
\begin{aligned}
r=-\lambda_{\min }^{-1}\left([\operatorname{diag}(b)]^{-1 / 2}[\right. & \operatorname{diag}(b) A \\
& \left.+A^{T} \operatorname{diag}(b)-b b^{T}\right] \\
& \left.\times[\operatorname{diag}(b)]^{-1 / 2}\right),
\end{aligned}
$$

where $\lambda_{\min }(\cdot)$ denotes the smallest eigenvalue of the matrix $(\cdot)$.

\section{Conclusions and Discussions}

In this study, we show that the Runge-Kutta methods with stepsize restrictions can preserve global and asymptotical stability of the continuous DDEs or NDDEs. An algebraically stable Runge-Kutta method with linear interpolation procedure for DDEs or NDDEs is globally stable and asymptotically stable. These results can be easily extended to the following equation with several delays:

$$
\begin{gathered}
\frac{d}{d t}\left[y(t)-\sum_{i=1}^{l} N_{i} y\left(t-\tau_{i}\right)\right] \\
=f\left(t, y, y\left(t-\tau_{1}\right), \ldots, y\left(t-\tau_{l}\right)\right), \quad t \geq 0, \\
y(t)=\psi(t), \quad t \leq 0,
\end{gathered}
$$

under the following assumption:

$$
\begin{gathered}
\operatorname{Re}\left\langle(y-z)-\sum_{i=1}^{l} N_{i}\left(y_{i}-z_{i}\right), f\left(t, y, y_{1}, \ldots, y_{N}\right)\right. \\
\left.\quad-f\left(t, z, z_{1}, \ldots, z_{N}\right)\right\rangle \\
\leq \alpha\|y-z\|^{2}+\sum_{i=1}^{N} \beta_{i}\left\|y_{i}-z_{i}\right\|^{2} \\
+\delta\left\|f\left(t, y, y_{1}, \ldots, y_{N}\right)-f\left(t, z, z_{1}, \ldots, z_{N}\right)\right\|^{2}
\end{gathered}
$$

where $\tau_{i}>0, i=1,2, \ldots, l, y_{i}=y\left(t-\tau_{i}\right)$, and $z_{i}=z\left(t-\tau_{i}\right)$. We do not list them here for the sake of brevity.

Moreover, the present results have certain instructive effect in numerical simulation. In the future, we hope to apply the results to some real-world problems, for example, reaction-diffusion dynamical systems with time delay [24] and non-Fickian delay reaction-diffusion equations $[25,29]$.

\section{Conflict of Interests}

The authors declare that there is no conflict of interests regarding the publication of this paper.

\section{Acknowledgment}

This work is supported by NSFC (Grant nos. 11201161, 11171125, and 91130003).

\section{References}

[1] A. Bellen and M. Zennaro, Numerical Methods for Delay Differential Equations, Clarendon Press, Oxford, UK, 2003.

[2] H. Brunner, Collocation Methods for Volterra Integral and Related Functional Differential Equations, Cambridge University Press, Cambridge, UK, 2004.

[3] W. H. Enright and H. Hayashi, "Convergence analysis of the solution of retarded and neutral delay differential equations by continuous numerical methods," SIAM Journal on Numerical Analysis, vol. 35, no. 2, pp. 572-585, 1998.

[4] A. Bellen, N. Guglielmi, and M. Zennaro, "Numerical stability of nonlinear delay differential equations of neutral type," Journal of Computational and Applied Mathematics, vol. 125, no. 1-2, pp. 251-263, 2000.

[5] R. Vermiglio and L. Torelli, "A stable numerical approach for implicit non-linear neutral delay differential equations," BIT Numerical Mathematics, vol. 43, no. 1, pp. 195-215, 2003.

[6] C. Zhang, "Nonlinear stability of natural Runge-Kutta methods for neutral delay differential equations," Journal of Computational Mathematics, vol. 20, no. 6, pp. 583-590, 2002.

[7] W. Wang, S. Li, and K. Su, "Nonlinear stability of Runge-Kutta methods for neutral delay differential equations," Journal of Computational and Applied Mathematics, vol. 214, no. 1, pp.175185, 2008.

[8] W. Wang, S. Li, and K. Su, "Nonlinear stability of general linear methods for neutral delay differential equations," Journal of Computational and Applied Mathematics, vol. 224, no. 2, pp. 592-601, 2009.

[9] A. H. Bhrawy, A. AlZahrani, D. Baleanu, and Y. Alhamed, "A modified generalized Laguerre-Gauss collocation method for fractional neutral functional differential equations on the halfline," Abstract and Applied Analysis, vol. 2014, Article ID 692193, 7 pages, 2014.

[10] A. H. Bhrawy and M. A. Alghamdi, "A shifted Jacobi-Gauss collocation scheme for solving fractional neutral functionaldifferential equations," Advances in Mathematical Physics, vol. 2014, Article ID 595848, 8 pages, 2014.

[11] A. H. Bhrawy, L. M. Assas, E. Tohidi, and M. A. Alghamdi, "A Legendre-Gauss collocation method for neutral functionaldifferential equations with proportional delays," Advances in Difference Equations, vol. 2013, article 63, 2013.

[12] E. H. Doha, A. H. Bhrawy, D. Baleanu, and R. M. Hafez, "A new Jacobi rational-Gauss collocation method for numerical solution of generalized pantograph equations," Applied Numerical Mathematics, vol. 77, pp. 43-54, 2014.

[13] E. H. Doha, D. Baleanu, A. H. Bhrawy, and R. M. Hafez, "A pseudospectral algorithm for solving multipantograph delay systems on a semi-infinite interval using legendre rational functions," Abstract and Applied Analysis, vol. 2014, Article ID 816473, 9 pages, 2014.

[14] D. Li and C. Zhang, "Nonlinear stability of discontinuous Galerkin methods for delay differential equations," Applied Mathematics Letters, vol. 23, no. 4, pp. 457-461, 2010.

[15] Y. L. Niu and C. J. Zhang, "Exponential stability of nonlinear delay differential equations with multidelays," Acta Mathematicae Applicatae Sinica, vol. 31, no. 4, pp. 654-662, 2008.

[16] L. Ferracina and M. N. Spijker, "Stepsize restrictions for totalvariation-boundedness in general Runge-Kutta procedures," Applied Numerical Mathematics, vol. 53, no. 2-4, pp. 265-279, 2005. 
[17] I. Higueras, "On strong stability preserving time discretization methods," Journal of Scientific Computing, vol. 21, no. 2, pp. $193-$ 223, 2004.

[18] M. N. Spijker, "Stepsize restrictions for stability of one-step methods in the numerical solution of initial value problems," Mathematics of Computation, vol. 45, no. 172, pp. 377-392, 1985.

[19] M. N. Spijker, "Stepsize conditions for general monotonicity in numerical initial value problems," SIAM Journal on Numerical Analysis, vol. 45, no. 3, pp. 1226-1245, 2007.

[20] C. Zhang and Y. He, "The extended one-leg methods for nonlinear neutral delay-integro-differential equations," Applied Numerical Mathematics, vol. 59, no. 6, pp. 1409-1418, 2009.

[21] L. Wen, S. Wang, and Y. Yu, "Dissipativity of Runge-Kutta methods for neutral delay integro-differential equations," Applied Mathematics and Computation, vol. 215, no. 2, pp. 583-590, 2009.

[22] K. Dekker and J. G. Verwer, Stability of Runge-Kutta Methods for Stiff Nonlinear Differential Equations, North-Holland Publishing, Amsterdam, The Netherlands, 1984.

[23] K. J. In 't Hout, "Stability analysis of Runge-Kutta methods for systems of delay differential equations," IMA Journal of Numerical Analysis, vol. 17, no. 1, pp. 17-27, 1997.

[24] D. Li, C. Zhang, and H. Qin, "LDG method for reactiondiffusion dynamical systems with time delay," Applied Mathematics and Computation, vol. 217, no. 22, pp. 9173-9181, 2011.

[25] D. Li, C. Zhang, and W. Wang, "Long time behavior of nonFickian delay reaction-diffusion equations," Nonlinear Analysis: Real World Applications, vol. 13, no. 3, pp. 1401-1415, 2012.

[26] C. Huang, H. Fu, S. Li, and G. Chen, "Nonlinear stability of general linear methods for delay differential equations," BIT Numerical Mathematics, vol. 42, no. 2, pp. 380-392, 2002.

[27] Y. K. Liu, "Numerical solution of implicit neutral functionaldifferential equations," SIAM Journal on Numerical Analysis, vol. 36, no. 2, pp. 516-528, 1999.

[28] I. Higueras, "Monotonicity for Runge-Kutta methods: inner product norms," Journal of Scientific Computing, vol. 24, no. 1, pp. 97-117, 2005.

[29] D. Li, C. Tong, and J. Wen, "Stability of exact and discrete energy for non-Fickian reaction-diffusion equations with a variable delay," Abstract and Applied Analysis, vol. 2014, Article ID 840573, 9 pages, 2014. 


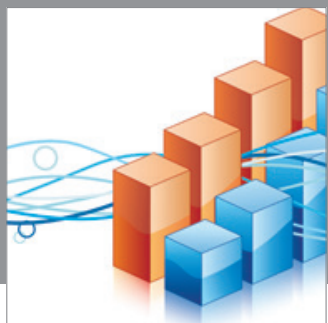

Advances in

Operations Research

mansans

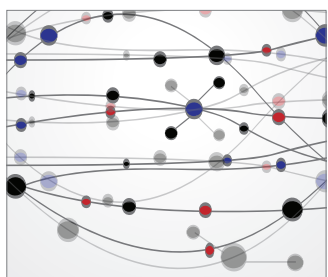

The Scientific World Journal
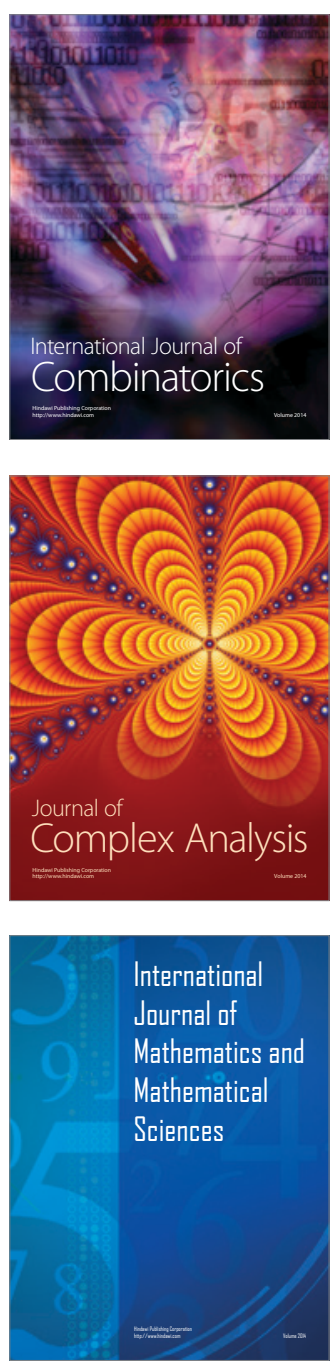
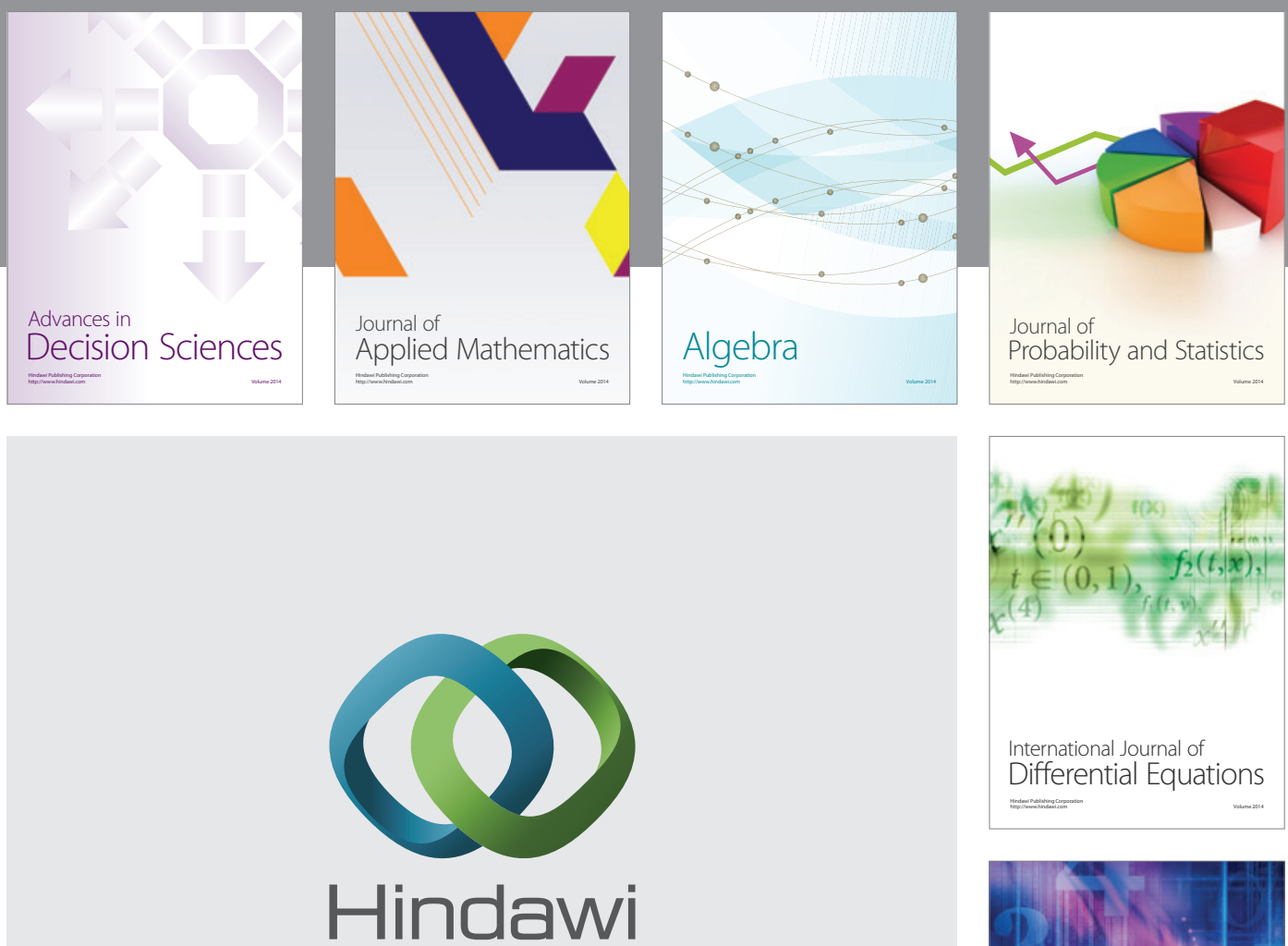

Submit your manuscripts at http://www.hindawi.com
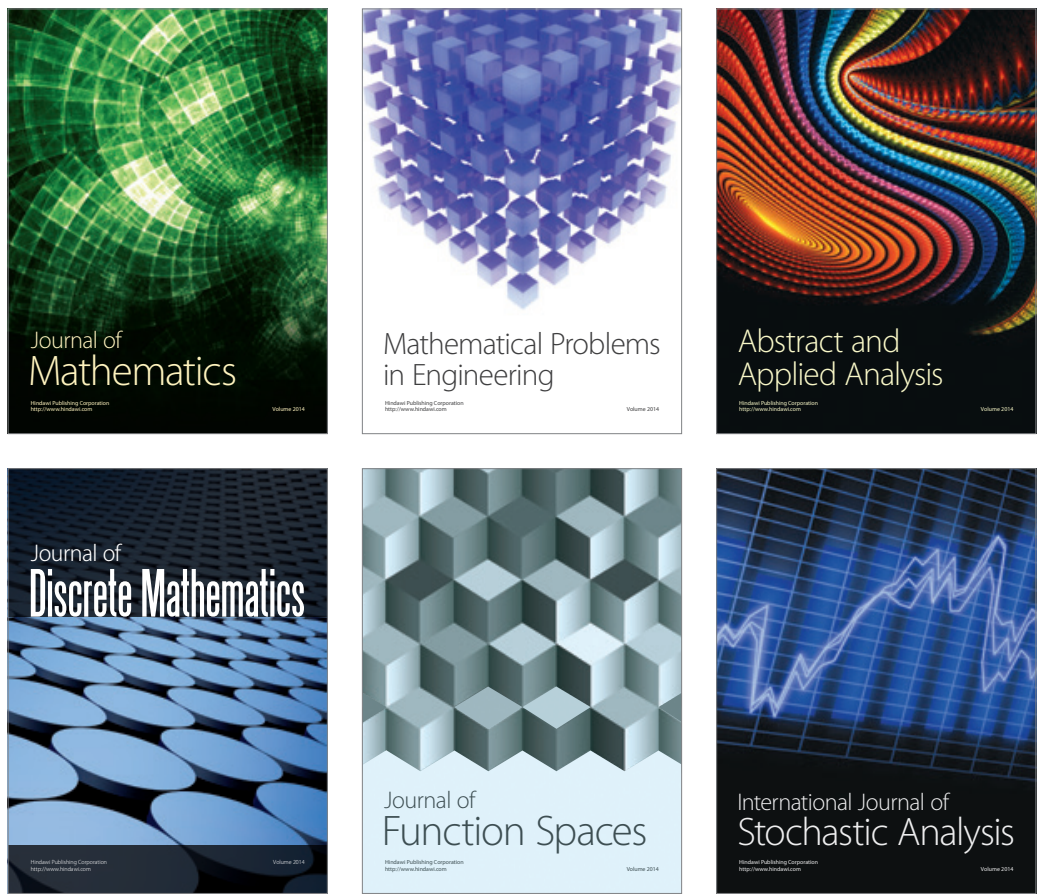

Journal of

Function Spaces

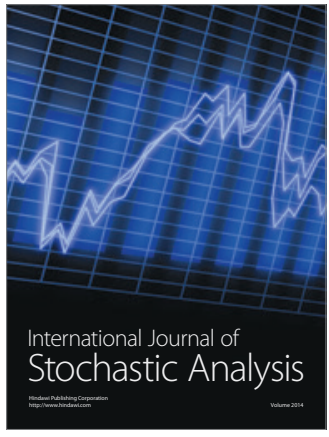

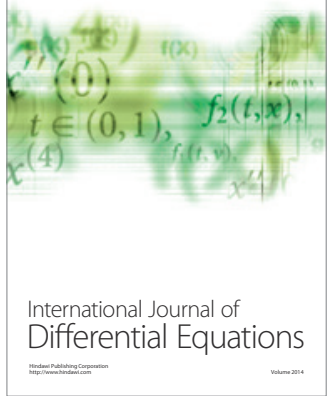
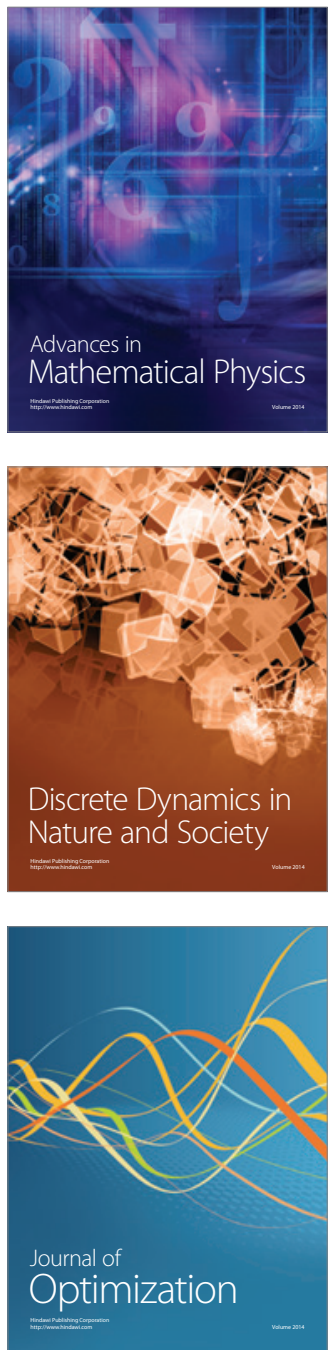